\title{
Predictors of Mortality
}

\section{Hospice Enrollment and Evaluation of Its Causal Effect on Hospitalization of Dying Nursing Home Patients}

\author{
Pedro L. Gozalo and Susan C. Miller
}

Objective. To examine the patient, nursing home (NH), hospice provider, and local market factors associated with the selection of the Medicare hospice benefit by eligible NH residents, and evaluate the causal effect of hospice on end-of-life hospitalization rates. Data Sources/Study Setting. Secondary data for 1995-1997 for NH residents.

Study Design. This retrospective cohort study includes NH residents in five states (Kansa, Maine, New York, Ohio, South Dakota) who died in the years 1995-1997. Medicare claims identified hospice enrollment and hospitalizations. Geocoding of NHs, hospice providers, and hospitals was used to identify local markets. The two outcome measures are hospice enrollment and hospitalization of $\mathrm{NH}$ residents in their last 30 days of life.

Data Collection/Extraction Method. A file was constructed linking MDS assessments to Medicare claims and denominator files, $\mathrm{NH}$ provider files (OSCAR), hospice provider of service files, and the area resource file.

Principal Findings. Twenty-six percent of hospice and 44 percent of nonhospice residents were hospitalized in their last 30 days of life (odds ratio [OR] 0.45; 95 percent confidence interval [CI]: 0.42-0.48). Adjusting for confounders, hospice patients were less likely than nonhospice residents to be hospitalized (OR 0.47 ; 95 percent CI: $0.45-$ 0.50). Adding inverse propensity score weighting, hospice patients were still less likely than nonhospice residents to be hospitalized (OR 0.56; 95 percent CI: 0.53-0.61).

Conclusions. Hospice selection introduces some bias in the evaluation of the causal effect of hospice on end-of-life hospitalization rates. However, even after adjusting for selection bias, hospice does have a powerful effect in reducing end-of-life hospitalization rates.

Key Words. Hospice care, MDS, terminal care, hospitalization rates, selection bias

During the last decade, the Medicare Hospice Benefit has become an integral part of the care for a large number of terminally ill people in the United States. From 1992 to 2000 the number of hospice beneficiaries almost quadrupled, from about 157,000 (or around 9 percent of decedents 65 years old or older) 11 percent of whom lived in a nursing home $(\mathrm{NH})$ - to nearly 535,000 people 
(about 30 percent of aged decedents) - 36 percent of whom lived in a NH (Centers for Medicare and Medicaid Services 2001; MedPAC 2002). The growth of NHs offering hospice to their residents has also been particularly large, increasing from an estimated 30 percent of U.S. NHs in 1995-1997 (Petrisek and Mor 1999) to 76 percent in 2000 (Miller and Mor 2004).

Hospice provides an alternative to aggressive curative care; benefits include better palliative treatment that puts emphasis on physical, emotional, and spiritual pain relief for the patient and immediate family members. To become eligible for hospice, a Medicare beneficiary needs to have been certified by his/her doctor (and the hospice medical director) as having a terminal prognosis of 6 months or less, if the terminal illness runs its expected course. Furthermore, choosing hospice entails forfeiting access to other Medicare Part A benefits, such as hospitalization and skilled nursing care, for treatment related to their terminal condition.

One of the main observed effects associated with hospice has been the lower hospitalization rates among home-based hospice (Greer et al. 1983; Mor and Kidder 1985; Kidder 1992; Lewin-VHI 1995). Evidence suggests that, when integrated into the $\mathrm{NH}$ care process, hospice care is also associated with lower rates of hospitalization for $\mathrm{NH}$ hospice patients. Additionally, nonhospice patients who died in $\mathrm{NHs}$ having a hospice presence have also been found to have lower rates of end-of-life hospitalizations (Miller, Gozalo, and Mor 2001). This reduction in hospitalization rates has two main implications. At the patient level, hospitalizations of frail $\mathrm{NH}$ residents have been shown to include hazards that negatively affect the quality of life (Creditor 1993) and, in many cases, are inappropriate (Saliba et al. 2000). At the policy level, hospitalizations represent the main component of total health care costs, particularly during the last few months of life. In an recent study using both Medicare and Medicaid claims for NH decedents in the state of Florida in 1999, Miller et al. (2004) found hospital expenditures to account on average for 78 percent of all expenditures in the last month of life among those patients that did not receive hospice and 33 percent among $\mathrm{NH}$ residents who had any hospice in the last 30 days of life. ${ }^{1}$

One important criticism of the above types of analyses is that selection bias is introduced by the nonrandom nature of the assignment to hospice, and

Address correspondence to Pedro L. Gozalo, Ph.D., M.Sc., Investigator, Center for Gerontology and Health Care Research, Department of Community Health, Brown University School of Medicine, Box G-ST211, 2 Stimson Street, Providence, RI 02912. Susan C. Miller, Ph.D., M.B.A., is with the Center for Gerontology and Health Care Research, Department of Community Health, Brown University School of Medicine, Providence, RI. 
when not adjusted for, results may be biased (Emanuel and Emanuel 1994, Scitovsky 1994). Patients that satisfy the hospice eligibility criteria (and are knowledgeable about the status of their condition and about the availability of Medicare hospice) are free to choose to participate in the benefit. As a result, individuals that choose to enroll in hospice may have opted-regardless of hospice-for less aggressive care at end-of-life. Ignoring this self-selection would wrongly ascribe to hospice the lower inherent propensity of those hospice recipients to be hospitalized. An additional source of selection bias for $\mathrm{NH}$ residents stems from the fact that NHs also "choose hospice"- choose to have in place a contractual agreement with a hospice provider. As with residents, nursing facilities offering hospice may differ in important ways in their use of aggressive care from those without a hospice contract. There is some evidence in the literature that point to the influence of facility and (local) market characteristics on hospice enrollment and end-of-life hospitalization. An evaluation of the timing of hospice enrollment found strong market and provider effects, even after adjusting for several patient clinical attributes (Christakis and Iwashyna 2000). Similarly, other studies have found local health care markets to be associated with site of death (Pritchard et al. 1998; Gallo, Baker, and Bradley 2001); in particular, one study found death in a hospital setting to be highly influenced by local health care system characteristics, more so than by patient characteristics and their preferences (Pritchard et al. 1998). Consequently, it is important to consider both individual characteristics, and facility and market characteristics when controlling for selection bias.

This study makes two substantial contributions to the literature. First, it estimates NH residents' propensity to elect hospice, which has not been previously examined in the literature. Second, and more importantly, this study uses inverse propensity score weighting to estimate the true (causal) hospice effect on hospitalization of $\mathrm{NH}$ residents in their last 30 days of life. We hypothesized that the hospice effect on hospitalization would be smaller once we controlled for residents' propensity to elect hospice, but that there would still be a large hospice effect.

\section{MeTHODS}

\section{Data Sources and Cohort Development}

Having obtained a signed data use agreement from the Center for Medicare and Medicaid Services (CMS), we linked all the Minimum Data Set (MDS) $\mathrm{NH}$ assessment records in five states (Kansas, Maine, New York, Ohio, and 
South Dakota) with the CMS eligibility file which contains the Medicare beneficiary date of death (DoD). We identified 225,254 NH residents in these states who died in the years $1995-1997 .^{2}$ We deleted 307 (0.14 percent) of these residents due to inconsistent or illogical data elements, and an additional 3,167 (1.41 percent) residents with no $\mathrm{NH}$ identification number. In addition, a total of 36,030 (16.0 percent) residents who had their last MDS assessment 120 days or more before their $\mathrm{DoD}$ were excluded as, given the requirement that MDS assessments be collected quarterly at the latest, it was unlikely that they were still $\mathrm{NH}$ residents at their time of death.

Using the unique personal identifier of the 185,750 remaining $\mathrm{NH}$ residents we linked their MDS records and their Medicare hospice and inpatient claims. Comparing their $\mathrm{NH}$ admission date(s) and the coverage periods of their hospice episodes we identified 14,615 (7.9 percent) residents who were enrolled in hospice while being a NH resident. An additional 2,008 (1.1 percent of total) who enrolled in hospice before their $\mathrm{NH}$ admission were also identified and excluded because they were not exposed to the usual care practices of the $\mathrm{NH}$ at the time of their hospice election and could potentially receive different treatment than residents who selected hospice after being admitted in the NH. The final cohort for our analysis consisted of 183,742 residents, 169,127 who did not enroll in hospice and the 14,615 (8 percent) who did. Residents identified as hospice enrollees could have been enrolled in hospice from 1 to 30 days in their last 30 days of life.

Organizational data on $\mathrm{NH}$ providers was obtained from the CMS Online Survey and Certification Automated Record (OSCAR). Additional CMS data files used in the analysis included the 1997 Provider of Services (POS) file. $\mathrm{NH}$, hospice and hospital provider addresses in these files were used to obtain geocoded variables, such as latitude and longitude, for these facilities. We also used the 1998 release of the Area Resource File (ARF).

\section{Analytic Approach}

Our main goal was to estimate the causal treatment effect of hospice on end-oflife hospitalization. In the absence of randomization, individuals choosing hospice will generally be different in observed and unobserved characteristics than those not choosing hospice. The two main approaches to estimate causal effects of nonrandomized treatment are methods based on controlling for observed differences (these include regression, matching, and propensity score) and those based on instrumental variables (IV). IV estimation relies on the identification of one (or more) variables, which affects treatment but that 
only affects the outcome through the treatment. The IV method has the potential for controlling unobserved confounding, but the validity of the IV itself cannot be empirically checked (Angrist, Imbens, and Rubin 1996; McClellan and Newhouse 2000). In our case, the identification of a variable which could satisfy the two requirements of affecting hospice enrollment by $\mathrm{NH}$ residents and at the same time not affecting hospitalization in their last 30 days of life (other than through the hospice enrollment) was not obvious. For this reason, our approach focused on a method that controls for observed characteristics.

We took advantage of the relative richness of our datasets to create a large set of observed individual characteristics to minimize the effects of potential unobserved factors. In particular, our estimator is based on the Inverse Probability of Treatment Weighting (IPTW) method proposed by Robins and colleagues (Robins and Rotnitzky 1995; Robins, Hernan, and Brumback 2000). The IPTW method combines two commonly used methods, regression and propensity score weighting, to take advantage of the best features of each individual method. Regression corrects for confounding while the inverse propensity score weighting corrects for imbalances in the distribution of the covariates between the treatment and control groups due to the nonrandomness of the hospice enrollment process. Weighting by the inverse of the probability of treatment balances the distributions of the covariates among treatment groups producing an unbiased treatment effect estimate (Hirano and Imbens 2001; Hogan and Lancaster 2004; Imbens 2004).

Letting $Y$ denote the (binary) outcome of hospitalization of $\mathrm{NH}$ residents in their last 30 days of life, we estimated the treatment effect of hospice $H$ on $Y$. $H$ took the value of 1 if the resident elects hospice before death, regardless of how close to death, and 0 otherwise. The two-step estimation approach consisted of first estimating $P(W)=P(H=1 \mid W)$, the probability of choosing $H$ given $W=(X, Z)$, where $X$ contains observed pretreatment covariates determining hospitalization, and $Z$ a set of hospice provider characteristics. To reduce endogeneity we used characteristics of the hospice provider nearest to the $\mathrm{NH}$ rather than those of the hospice used by the patient in the NH. In the second step we regress $Y$ on $H$ and $X$ weighting each individual's observation by $[H / P(W)]+[(1-H) /(1-P(W))]$, the inverse probability of receiving the treatment actually received (Horvitz and Thompson, 1952; Robins, Hernan, and Brumback 2000). Subjects choosing hospice $(H=1)$ were weighted by the inverse of $P(W)$ and untreated subjects by the inverse of $1-P(W){ }^{3,4}$ In order to examine the sensitivity of our results to unobserved characteristics, we used increasing levels of observed confounders. In particular, we modeled 
$Y$ as a function of $H$ alone, and of $H$ and $X$ with $X=(R), X=(R, F)$, and $X=(R, F, M)$, where $R$ denotes resident characteristics, $F$ nursing facility characteristics, and $M$ local health market characteristics.

Analysis of the binary dependent variables in each of the two steps, $H$ and $Y$, was performed by logistic regression using the generalized estimating equation (GEE) method with robust standard errors that accounts for correlation in the error term due to clustering of individuals in the same nursing facility assuming an exchangeable working correlation (Liang and Zeger 1986; Diggle et al. 2002).

\section{Patient-Level Variables}

To carry out our analysis we collected two MDS assessments for each NH resident. For estimation of the probability of hospice treatment (step 1) we considered the assessment closest to the start of the hospice eligibility period of 6 months before death. For the approximately 33 percent of patients that were not admitted to the $\mathrm{NH}$ before their last 6 months of life, we used their $\mathrm{NH}$ admission assessment. For estimation of the end-of-life hospitalization (second step), we selected the last assessment (closest to death) for being the most informative about the patient's health status near death.

We obtained the patient's gender, age, race, and date of death from the CMS eligibility and vital status file. Race was dichotomized as "nonwhite" or "white." To allow for nonlinearities, age was categorized into five groups$<75,75-79,80-84,85-89$, and $90 \pm$ of approximately equal sample sizes. Other variables that were categorized for similar concerns were distances between providers and time from MDS to DoD. We used the MDS assessments and CMS inpatient claims (from January 1992) to obtain diagnosis information. Diagnoses were grouped into one of four broad categoriescancer with no dementia/Alzheimer's disease, cancer with dementia/Alzheimer's disease, dementia/Alzheimer's disease without cancer, and diagnosis other than cancer or dementia/Alzheimer's disease. ${ }^{5}$

From the MDS dataset and its derived variables we obtained the marital status (married, not married), the presence of congestive heart failure or chronic obstructive pulmonary disease, a 6-point activities of daily living (ADL) index, the Cognitive Performance Scale (CPS) (Morris et al. 1994), and the presence of do-not-hospitalize (DNH) and do-not-resuscitate (DNR) orders. DNH and DNR orders have been documented to be independently associated with reduced hospitalization (Mor et al. 1997; Dobalian 2004) and to function as a marker for undocumented care limitations or as a mandate 
to limit care in general (Zweig et al. 2004). The ADL index ranges from 0 (minimum oversight) to 5 (highly dependent). The CPS scores range from 0 (intact) to 6 (very severe impairment). The $\mathrm{DNH}$ and DNR variables were included to adjust for unobserved patient preferences toward aggressive care. While hospices may require DNR orders our MDS variable is recorded independently of hospice status. Also, to control for patient preferences and disease severity in the two estimation steps, we included the number of hospitalizations during the 6 months before the date of the MDS assessment. Last, an indicator variable for short-stay $\mathrm{NH}$ patients (stays of $<90$ days) and the number of days from the MDS to death provided additional information on unobserved factors contributing to death.

\section{Provider-Level Variables}

Each of the two MDS assessments used in the analysis was matched to the closest OSCAR survey to obtain the following $\mathrm{NH}$ attributes: payer types (percent residents Medicaid, Medicare or private pay), proprietary status (forprofit versus not-for-profit), multifacility status (part of a chain versus not part of a chain), provider type (operated by hospital versus not operated by a hospital), presence of any type of special care unit (such as Alzheimer's), and number of health deficiency citations. In addition, we used MDS records from all $\mathrm{NH}$ residents (not just decedents) in each facility to calculate the yearly average nursing case-mix index (CMI) for each facility, as well as the percent of nonwhite residents in each facility. This CMI measures the severity and level of resources used; it is based on the Resource Utilization Groups (RUG) scale and it is scaled to a value of 1 for the average resident (Fries et al. 1994). $\mathrm{NH}$ addresses in OSCAR and hospice provider addresses in the POS file were used to obtain latitude and longitude geocoding information and to compute distances between the patient's $\mathrm{NH}$ and the hospice providers. Each $\mathrm{NH}$ resident record was then linked to the hospice provider data of the provider nearest in distance to the resident's NH. From the hospice POS file we selected the following hospice attributes: proprietary status (for-profit versus not-forprofit), provider type (operated by hospital, operated by skilled nursing facility, operated by government agency, operated by home health agency, and free-standing hospice program), and type of care provided (inpatient care or respite care).

Finally, based on preliminary analysis, we included interaction terms for hospice for-profit proprietary status and both $\mathrm{NH}$ for-profit proprietary status and being part of a chain status. 


\section{Market and Environmental Variables}

For the hospice selection model, we constructed three variables to account for hospice market competition using distance from the $\mathrm{NH}$ to the nearest hospice provider, distance from the $\mathrm{NH}$ to the second nearest hospice provider, and an indicator of the presence of two or more hospice providers within 10 miles from the NH. Similarly, we constructed the distance from the $\mathrm{NH}$ to the nearest hospital and its number of beds, and an indicator of two or more hospitals within 10 miles of the $\mathrm{NH}$. To account for local health care market variation, we used the $\mathrm{ARF}$ data for the county of residence of each $\mathrm{NH}$ to determine the number of hospital beds and doctors per 100 persons 65 years of age or older. In addition, we used OSCAR data to calculate the number of $\mathrm{NH}$ beds per 100 persons 65 years of age or older in each county. We also included indicator variables for the five states.

\section{RESULTS}

\section{Characteristics of Patients}

A summary of the attributes of the hospice and nonhospice members of the cohort at the time of their last MDS assessment, their NH and hospice providers, and their local health care market is provided in Table 1. Hospice patients were different from nonhospice patients in some aspects. They were less often male and nonwhite and more often married. Hospice patients were 2 years younger on average than were nonhospice patients, but these differences were not statistically significant. As anticipated, a significantly higher proportion of hospice patients had documented DNH and DNR orders and had diagnoses of cancer (47.4 versus 22.4 percent; Table 1). The most common types of cancer among hospice and nonhospice patients were prostate (10.2 versus 14.2 percent of all cancer diagnoses), lung (15.4 versus 12 percent), colon (10 versus 9.7 percent), breast (8.2 versus 7.9 percent), and bladder (4 versus 4.8 percent), and almost half of the cancer patients had some metastasis (48 versus 42 percent) as secondary diagnosis (Cooper et al. 1999).

Hospice $\mathrm{NH}$ residents more often than nonhospice residents are in $\mathrm{NHs}$ that are for-profit, part of a chain, that have a higher private-pay/Medicaid-pay ratio, that have a lower percentage of nonwhite residents, that have a special care unit, and whose nearest hospice provider is for-profit and/or hospital based (Table 1). Compared with nonhospice residents, hospice residents are in NHs that are near a greater number of hospitals, closer to a large hospital, 2 miles closer on average to their closest hospice provider and 4 miles closer to 


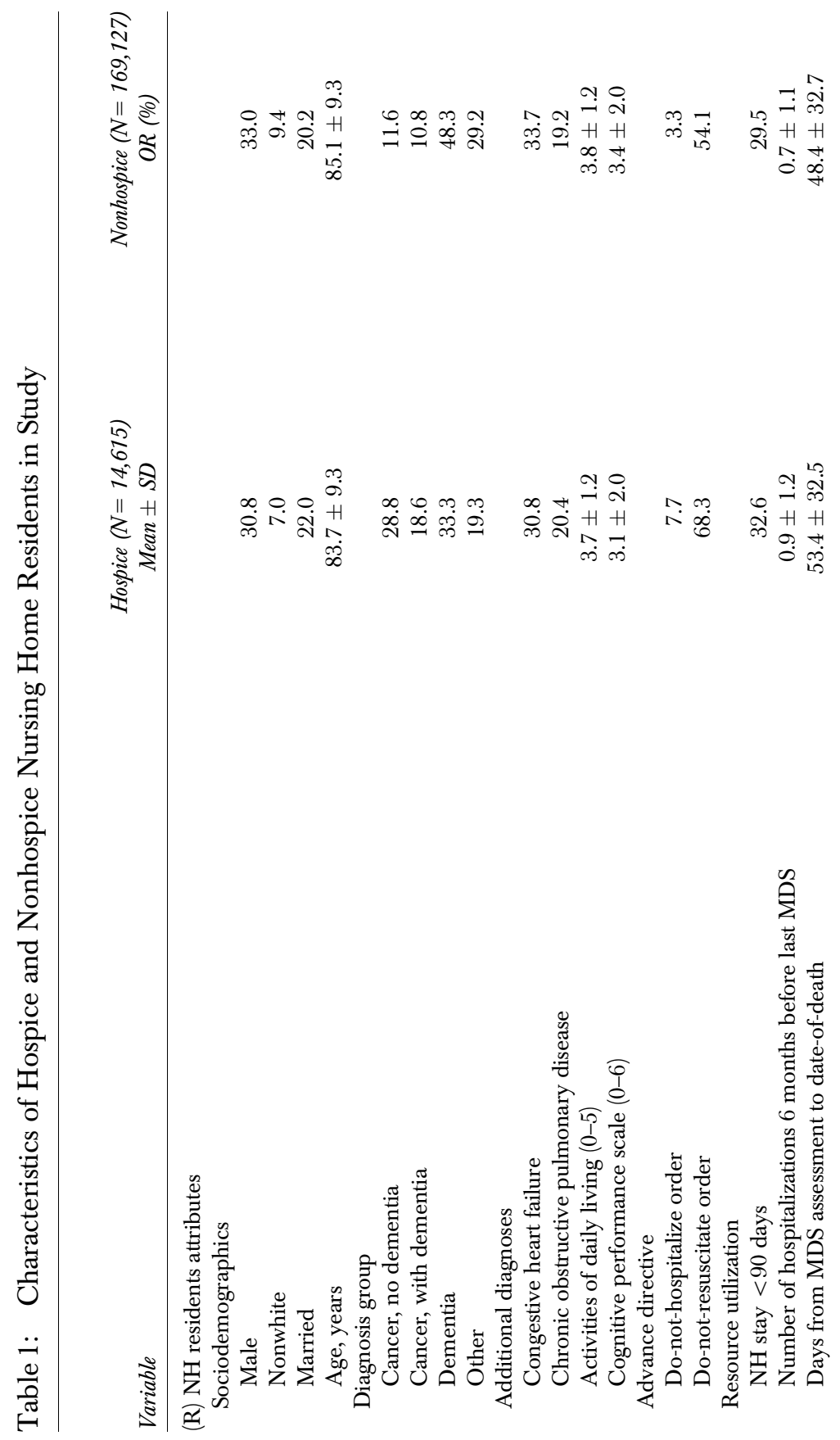




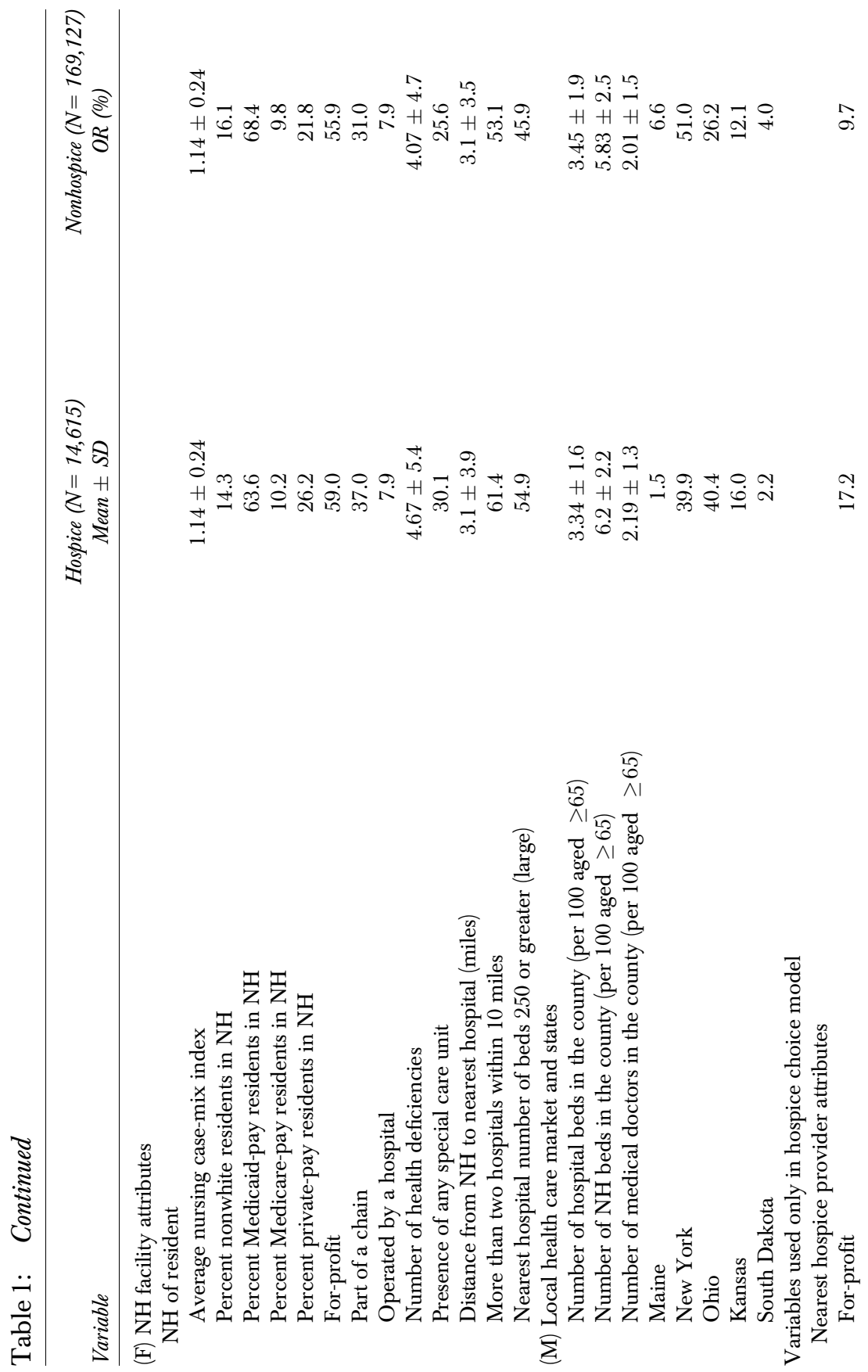




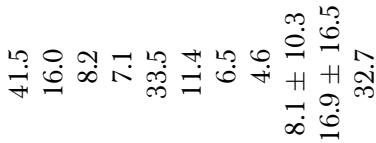

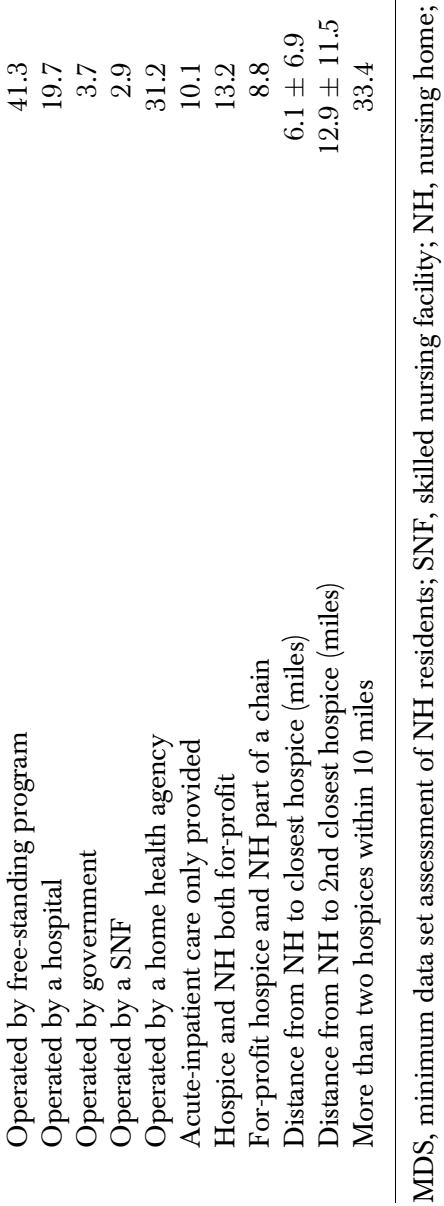


the second closest hospice provider. Finally, hospice enrollment rates vary substantially among the five states; rates were 12.5 percent in Ohio, 11.6 percent in Kansas, 6.6 percent in New York, 5.4 percent in South Dakota, and 2.4 percent in Maine.

\section{Factors Associated with the Choice of Hospice}

Table 2 shows the results of a logistic GEE multivariate regression of hospice enrollment of $\mathrm{NH}$ patients. The most important individual determinants of hospice enrollment are a principal diagnosis of cancer and preferences for noncurative interventions care as measured by the presence of a DNR and $\mathrm{DNH}$ order. Other patient factors significantly associated with higher hospice enrollment are being married and having a higher number of hospitalizations 6 months before the MDS assessment. Being male, nonwhite, older, having congestive heart failure and having higher physical and cognitive impairment were associated with a lower probability of hospice enrollment.

Among NH attributes, residents in facilities with higher average nursing CMI or with a special care unit had a greater likelihood of choosing hospice while residents in facilities with higher percentage of nonwhites were less likely to choose hospice. Providers' chain and for-profit status and having a high number of hospitals near the $\mathrm{NH}$, which appeared associated with higher hospice enrollment in descriptive analysis (Table 1), were not significantly associated with hospice enrollment. However, when the nearest hospital is large, or when both the $\mathrm{NH}$ and its nearest hospice provider were for-profit, residents had a higher likelihood of enrolling in hospice.

Distance between the $\mathrm{NH}$ and its nearest hospice provider in excess of 15 miles was significantly associated with a lower likelihood of hospice enrollment as was the nearest hospice provider being operated by a skilled nursing facility, government agency or a home health agency, or when only offering inpatient care. Distance to the nearest hospital or having many hospitals nearby, on the other hand, were not associated with higher hospice enrollment. Our adjustments for patient, facility, and local market characteristics still leave significant variations across states; $\mathrm{NH}$ patients in Ohio and Kansas had an over four times greater likelihood of choosing hospice than in Maine.

An analysis of the predicted probabilities of hospice enrollment used to construct the inverse probability weights for the estimation of end-of-life hospitalization showed the mean $\pm \mathrm{SD}$ of the predicted probabilities of hospice enrollment was $0.07 \pm 0.06$ for patients that did not actually enroll in hospice 
Table 2: Factors Associated with Hospice Enrollment of Nursing Home Patients*

Variable

OR $(95 \% C I)$

(R) NH residents attributes

Sociodemographics

Male

Nonwhite

$0.80(0.77,0.84)^{\ddagger}$

Married

$0.90(0.83,0.97)^{\ddagger}$

Age, per year

$1.13(1.08,1.18)^{\ddagger}$

$0.99(0.98,0.99)^{\ddagger}$

Diagnosis group

Other

1.00

Cancer, no dementia

$3.21(3.03,3.41)^{\ddagger}$

Cancer, with dementia

$2.29(2.14,2.46)^{\ddagger}$

Dementia

$1.05(1.00,1.11)^{\dagger}$

Additional diagnoses

Congestive heart failure

Chronic obstructive pulmonary disease

$0.89(0.85,0.93)^{\ddagger}$

$0.99(0.94,1.04)$

Activities of daily living (per unit increase)

$0.97(0.95,0.99)^{\dagger}$

Cognitive performance scale (per unit increase)

$0.95(0.94,0.96)^{\ddagger}$

Advance directive

Do-not-hospitalize order

$1.34(1.21,1.49)^{\ddagger}$

Do-not-resuscitate order

$1.30(1.24,1.36)^{\ddagger}$

Resource utilization

$\mathrm{NH}$ stay $<90$ days

$1.03(0.98,1.09)$

Number of hospitalizations 6 months before hospice MDS

$1.08(1.06,1.09)^{\ddagger}$

Days from MDS assessment to date-of-death (months)

$$
\begin{aligned}
& <3 \\
& 3 \leq 6 \\
& 6+
\end{aligned}
$$

1.00

$1.59(1.49,1.70)^{\ddagger}$

$1.49(1.39,1.60)^{\ddagger}$

(F) NH facility attributes

$\mathrm{NH}$ of patient

Average nursing case-mix index (per 0.1 units increase)

Percent nonwhite residents in $\mathrm{NH}$ (per 1\% increase)

Percent Medicaid-pay residents in NH (per 10\% increase)

Percent Medicare-pay residents in NH (per 10\% increase)

$0.65(0.44,0.95)^{\dagger}$

1.00

Percent private-pay residents in $\mathrm{NH}$ (per 10\% increase)

$1.02(0.98,1.06)$

$1.03(1.00,1.06)^{\dagger}$

For-profit

$1.01(0.87,1.18)$

Part of a chain

$1.09(0.96,1.23)$

Operated by a hospital

$0.98(0.76,1.23)$

$1.00(0.99,1.00)$

$1.10(0.99,1.22)^{\dagger}$

Presence of any special care unit

1.00

$$
\begin{aligned}
& <2 \\
& 2 \leq 6 \\
& 6+
\end{aligned}
$$

$1.09(0.96,1.24)$

$1.02(0.88,1.19)$

Nearest hospital size is large (250+ beds)

$1.21(1.02,1.43)^{\dagger}$

$1.03(0.87,1.23)$

More than two hospitals within 10 miles 
Table 2: Continued

\begin{tabular}{ll}
\hline Variable & OR $(95 \%$ CI) \\
\hline Nearest hospice provider & \\
For-profit & $0.96(0.75,1.23)$ \\
Operated by a hospital & 1.00 \\
Operated by free-standing program & $0.91(0.78,1.06)$ \\
Operated by a home health agency & $0.79(0.67,0.93)^{\ddagger}$ \\
Operated by government & $0.69(0.55,0.86)^{\ddagger}$ \\
Operated by a SNF & $0.51(0.38,0.70)^{\ddagger}$ \\
Respite care provided & 1.00 \\
Acute/inpatient care only provided & $0.78(0.66,0.92)^{\ddagger}$ \\
Hospice and NHH both for-profit & $1.58(1.23,2.02)^{\ddagger}$ \\
For-profit hospice and NHH part of a chain & $1.03(0.82,1.30)$ \\
Distance from NH to closest hospice (miles) & \\
$\quad<15$ & 1.00 \\
$15 \leq 30$ & $0.88(0.72,1.07)$ \\
$30+$ & $0.49(0.32,0.73)^{\ddagger}$ \\
Additional distance from NH to second closest hospice (miles) & \\
$\quad<15$ & 1.00 \\
$15 \leq 30$ & $1.06(0.89,1.26)$ \\
$30+$ & $0.90(0.67,1.20)$ \\
More than two hospices within 10 miles & $0.98(0.82,1.17)$ \\
(M) Local health care market and states & \\
Number of hospital beds in the county (per 100 aged $\geq 65)$ & $0.96(0.93,1.00)$ \\
Number of NH beds in the county (per 100 aged $\geq 65)$ & $1.05(1.03,1.07)^{\ddagger}$ \\
Number of medical doctors in the county (per 100 aged $\geq 65)$ & $1.13(1.06,1.21)^{\ddagger}$ \\
Maine & 1.00 \\
New York & $2.23(1.60,3.11)^{\ddagger}$ \\
Ohio & $4.51(3.29,6.18)^{\ddagger}$ \\
Kansas & $4.16(2.97,5.82)^{\ddagger}$ \\
South Dakota & $2.18(1.35,3.50)^{\ddagger}$ \\
\hline
\end{tabular}

*The table shows the results of a generalized estimating equation logistic regression giving the ORs and 95\% CIs of electing Medicare's hospice depending on patient, $\mathrm{NH}$, hospice provider, local health market, and state attributes in a sample of 183,742 $\mathrm{NH}$ residents. CIs were adjusted to account for natural clustering of patients into NHs. All dichotomous variables were coded as $1=$ present and $0=$ absent. Reference categories are denoted with an OR of 1.00 and no $95 \%$ CIs. ${ }^{\dagger} p<.05$; ${ }^{\ddagger} p<.001$.

MDS, minimum data set assessment of $\mathrm{NH}$ residents; SNF, skilled nursing facility; $\mathrm{NH}$, nursing home; OR, odds ratio; CI, confidence interval.

and $0.13 \pm 0.09$ for those that went on to choose hospice. The median predicted probabilities were 0.05 and 0.1 , respectively.

Factors Associated with Hospitalization in the Last 30 Days of Life

In the 30 days before death, 25 percent $(3,730)$ of the hospice patients and 43 percent $(73,410)$ of the nonhospice patients were hospitalized. ${ }^{6}$ This translates 
into a crude (unadjusted) odds ratio (OR) of 0.45 and a crude risk ratio (RR) of 0.59. ${ }^{7}$ Table 3 presents the estimated hospice effect on end-of-life hospitalization without and with adjustment based on the IPTW weights. The results are shown using increasing sets of covariates to better understand the effect of adjusting for different sets of observable confounders directly through the regression. Considering the results without IPTW adjustment, we see a moderate effect of adjusting for individual characteristics (OR 0.48; 95 percent confidence interval [CI]:0.45-0.51. RR 0.62; 95 percent CI: 0.59-0.64), and there was little change when facility and market factors were added. Adjusting with the IPTW weights based on the inverse probability of hospice treatment results in a significant adjustment in the model with no other covariates $(\mathrm{OR}$ 0.55 ; 95 percent CI: $0.51-0.59$; RR: 0.68 ; 95 percent CI: $0.65-0.72$ ), pointing to the presence of selection bias arising from differences in the distribution of the observed confounders between hospice and nonhospice $\mathrm{NH}$ residents, and very little additional effect when $\mathrm{NH}$ resident, facility, and market factors were added to this model. ${ }^{8}$ Comparing the crude RR (0.59) and fully adjusted $\mathrm{RR}(0.69)$ we see that of the 41 percent reduction in hospitalizations attributed to hospice by the crude RR, almost a quarter $([0.41-0.31] / 0.41=24.4$ percent) of them are estimated by our adjusted RR to be due to selection.

Table 4 presents the detailed results of the model adjusting for all groups of covariates with IPTW weighting adjustment. Individual factors significantly

Table 3: Effect of Hospice on End-of-Life Hospitalization in Models with Increasing Sets of Covariates*

\begin{tabular}{lccccc}
\hline & \multicolumn{2}{c}{ Without IPT Weighting } & & \multicolumn{2}{c}{ With IPT Weighting } \\
\cline { 2 - 3 } \cline { 6 - 7 } Covariates in the Model & OR (95\% CI) & RR (95\% CI) & & OR (95\% CI) & RR (95\% CI) \\
\hline H & $0.45(0.42,0.48)$ & $0.59(0.57,0.61)$ & & $0.55(0.51,0.59)$ & $0.68(0.65,0.72)$ \\
H, R & $0.48(0.45,0.51)$ & $0.62(0.59,0.64)$ & & $0.56(0.52,0.61)$ & $0.69(0.66,0.73)$ \\
H, R, F & $0.47(0.45,0.50)$ & $0.61(0.59,0.64)$ & & $0.56(0.52,0.60)$ & $0.69(0.66,0.73)$ \\
H, R, F, M & $0.47(0.45,0.50)$ & $0.61(0.59,0.64)$ & & $0.56(0.53,0.61)$ & $0.69(0.66,0.73)$ \\
\hline
\end{tabular}

*The table shows the results of a generalized estimating equation logistic regression giving the odds ratios (OR) and risk ratios (RR; with their 95\% CIs) of hospitalization in the last 30 days of life depending on hospice enrollment $(\mathrm{H}), \mathrm{NH}$ resident $(\mathrm{R}), \mathrm{NH}$ facility $(\mathrm{F})$, local health market and state $(\mathrm{M})$ attributes, in a sample of $183,742 \mathrm{NH}$ residents (see Table 4 for detailed list of variables used). Robust confidence intervals were adjusted to account for natural clustering of patients into NHs. The adjusted results used stabilized weights proportional to the inverse probability of receiving the (hospice) treatment actually received. These probabilities of electing hospice were computed for all models using the results displayed in Table 2. All hospice coefficient estimates have a $p$-value $<.001$.

$\mathrm{NH}$, nursing home; $\mathrm{CI}$, confidence interval. 
Table 4: Effect of Hospice and Full Set of Factors on End-of-Life Hospitalization (Using IPT Weighting)*

\begin{tabular}{|c|c|}
\hline Variable & Odds Ratio (95\% CI) \\
\hline Hospice enrollment after $\mathrm{NH}$ admission & $0.56(0.53,0.61)^{\ddagger}$ \\
\hline \multicolumn{2}{|l|}{ (R) NH residents attributes } \\
\hline \multicolumn{2}{|l|}{ Resident sociodemographics } \\
\hline Male & $1.07(1.05,1.10)^{\ddagger}$ \\
\hline Nonwhite & $0.96(0.91,1.00)^{\dagger}$ \\
\hline Married & $1.06(1.03,1.09)^{\ddagger}$ \\
\hline \multicolumn{2}{|l|}{ Age (years) } \\
\hline$<75$ & 1.00 \\
\hline $75 \leq 80$ & $1.03(0.99,1.07)$ \\
\hline $80 \leq 85$ & $0.99(0.95,1.03)$ \\
\hline $85 \leq 90$ & $0.95(0.91,0.98)^{\ddagger}$ \\
\hline $90+$ & $0.78(0.74,0.81)^{\ddagger}$ \\
\hline \multicolumn{2}{|l|}{ Patient diagnosis group } \\
\hline Other & 1.00 \\
\hline Cancer, no dementia & $0.91(0.88,0.94)^{\ddagger}$ \\
\hline Cancer, with dementia & $1.14(1.10,1.18)^{\ddagger}$ \\
\hline Dementia & $1.11(1.08,1.14)^{\ddagger}$ \\
\hline \multicolumn{2}{|l|}{ Additional diagnoses } \\
\hline Congestive heart failure & $1.15(1.12,1.17)^{\ddagger}$ \\
\hline Chronic obstructive pulmonary disease & $1.08(1.05,1.11)^{\ddagger}$ \\
\hline Activities of daily living (per unit increase) & $0.88(0.87,0.89)^{\ddagger}$ \\
\hline Cognitive performance scale (per unit increase) & $0.92(0.91,0.93)^{\ddagger}$ \\
\hline \multicolumn{2}{|l|}{ Advance directive } \\
\hline Do-not-hospitalize order & $0.60(0.56,0.64)^{\ddagger}$ \\
\hline Do-not-resuscitate order & $0.70(0.68,0.71)^{\ddagger}$ \\
\hline \multicolumn{2}{|l|}{ Resource utilization } \\
\hline $\mathrm{NH}$ stay $<90$ days & $1.47(1.42,1.52)^{\ddagger}$ \\
\hline Number of hospitalizations 6 months before last MDS & $1.15(1.14,1.17)^{\ddagger}$ \\
\hline \multicolumn{2}{|l|}{ Days from MDS assessment to date-of death (days) } \\
\hline$\leq 21$ & 1.00 \\
\hline $2 \overline{2}-90$ & $0.37(0.36,0.38)^{\ddagger}$ \\
\hline $91+$ & $0.47(0.44,0.49)^{\ddagger}$ \\
\hline \multicolumn{2}{|l|}{ (F) $\mathrm{NH}$ attributes } \\
\hline Average nursing case-mix index (per 0.1 units increase) & $1.01(1.00,1.02)^{\dagger}$ \\
\hline Percent nonwhite residents in $\mathrm{NH}$ (per $1 \%$ increase) & $2.28(1.93,2.68)^{\ddagger}$ \\
\hline Percent Medicaid-pay residents in NH (per $10 \%$ increase) & 1.00 \\
\hline Percent Medicare-pay residents in NH (per $10 \%$ increase) & $1.01(0.99,1.03)$ \\
\hline Percent private-pay residents in $\mathrm{NH}$ (per $10 \%$ increase) & $0.95(0.93,0.96)^{\ddagger}$ \\
\hline For-profit & $1.24(1.17,1.32)^{\ddagger}$ \\
\hline Part of a chain & $0.93(0.88,0.97)^{\ddagger}$ \\
\hline Operated by a hospital & $0.90(0.80,1.00)$ \\
\hline Number of health deficiencies (per unit increase) & $1.00(0.99,100)$ \\
\hline Presence of any special care unit & $0.97(0.92,1.01)$ \\
\hline
\end{tabular}




\section{Table 4: Continued}

\begin{tabular}{ll}
\hline Variable & Odds Ratio (95\% CI) \\
\hline Distance from NH to nearest hospital (miles) & 1.00 \\
$\quad<2$ & $0.93(0.87,0.99)^{\dagger}$ \\
2 to \& $<6$ & $0.95(0.88,1.01)$ \\
$6+$ & $0.99(0.90,1.07)$ \\
$\quad$ Nearest hospital size is large $(250+$ beds) & $1.31(1.22,1.41)^{\ddagger}$ \\
$\quad$ More than 2 hospitals within 10 miles & \\
(M) Local health care market and states & $0.99(0.98,1.01)$ \\
Number of hospital beds in the county (per 100 aged $\geq 65)$ & $1.00(0.99,1.01)$ \\
Number of NH beds in the county (per 100 aged $\geq 65)$ & $0.99(0.96,1.02)$ \\
Number of medical doctors in the county (per 100 aged $\geq 65)$ & 1.00 \\
Maine & $1.36(1.20,1.53)^{\ddagger}$ \\
New York & $1.28(1.13,1.45)^{\ddagger}$ \\
Ohio & $1.55(1.35,1.77)^{\ddagger}$ \\
Kansas & $1.56(1.32,1.84)^{\ddagger}$ \\
South Dakota &
\end{tabular}

*The table shows the results of a generalized estimating equation logistic regression giving the odds ratios and 95\% CIs of hospitalization in the last 30 days of life depending on hospice enrollment, patient, $\mathrm{NH}$, local health market, and state attributes in a sample of 183,742 NH residents. Robust confidence intervals were adjusted to account for natural clustering of patients into NHs assuming an exchangeable working correlation. The adjusted results used stabilized weights proportional to the inverse probability of receiving the (hospice) treatment actually received. These probabilities of electing hospice were computed using the results displayed in Table 2. All dichotomous variables were coded as $1=$ present and $0=$ absent. Reference categories are denoted with an odds ratio of 1.00 and no $95 \%$ CIs.

${ }^{\dagger} p<.05$;

${ }^{\ddagger} p<.001$.

$\mathrm{NH}$, nursing home; OR, odds ratio; CI, confidence interval.

associated with a lower likelihood of hospitalization include being nonwhite, older age, a diagnosis of cancer without dementia, greater functional limitations (ADL), and cognitive impairment (CPS), and the presence of a DNH or DNR order. ${ }^{9}$ Being male, married, having a diagnosis of dementia (with or without cancer), congestive heart failure or chronic obstructive pulmonary disease, being a short-stay $\mathrm{NH}$ resident and having been hospitalized more often in the 6 months before the MDS assessment date were all significantly associated with higher likelihood of hospitalization. Some $\mathrm{NH}$ attributes were significantly associated with hospitalization in the last 30 days of life, particularly the organizational characteristics, the proportion of nonwhite residents, being more than 2 miles away from the nearest hospital, and being near many hospitals. 


\section{DISCUSSION}

This study is the first to attempt to disentangle the causal hospice influence on end-of-life hospitalization from the influence that may be more correctly attributable to factors associated with hospice selection. Persons who enroll in hospice choose aggressive palliative care rather than aggressive curative care and, a logical assumption is that these same persons would also utilize less inpatient care, even if hospice was not available. Similarly, $\mathrm{NHs}$ that choose to contract with hospices may be less likely to hospitalize their residents, even if hospice was not present. Our results found that, indeed, about one quarter of the observed raw hospice effect on hospitalization in the last 30 days of life is eliminated when we use the IPTW method to adjust for selection on observable characteristics (from a RR of 0.59 to 0.69 ). However, the hospice effect still represents an important reduction in the likelihood of end-of-life hospitalizations, and this effect may have been even larger if we had not treated all hospice enrollees similarly (regardless of hospice length of stay). Therefore, the hypothesis that hospice enrollment is associated with a lower likelihood of end-of-life hospitalization is strongly supported by this research.

As Medicare hospice enrollees cannot access Medicare Part-A inpatient care for conditions related to their terminal illnesses (and remain enrolled in hospice) and because hospice is responsible for the care coordination of $\mathrm{NH}$ hospice residents, most NHs have procedures in place to notify hospice regarding the potential need for hospitalization. Once notified, hospice staff intervenes in an attempt to prevent hospitalization. Similar routine intervention does not occur for many nonhospice residents who become acutely ill, or are actively dying (Kayser-Jones et al. 1989; Brooks et al. 1994). So, in addition to the palliative care expertise that accompanies hospice enrollment, care protocols associated with hospice enrollment appear to enable the avoidance of end-of-life hospitalization.

As in previous research (Miller and Mor 2002), we found residents who enrolled in hospice more often had cancer diagnoses and DNR and DNH orders in place and that hospice enrollment varied tremendously across states. Only 2 percent of Maine, 5 percent of South Dakota, and 7 percent of New York NH dying residents accessed hospice, compared with 12 percent of Kansas and 13 percent of Ohio NH residents. These observed enrollment differences are compatible with overall hospice enrollment differences in these states (Gage and Dao 2000). As would be expected, residents who enrolled in hospice versus nonhospice dying residents, were in NHs that were closer in distance to the nearest hospice. 
In both multivariate models, the associations observed between end-oflife hospitalization and patient level variables (including the state in which patients resided) were consistent with previous research (Miller, Gozalo, and Mor 2001). Additionally, consistent with previous research (Intrator, Castle, and Mor 1999; Mor, Papandonatos, and Miller 2005), we found residents in for-profit NHs and in NHs with higher proportions of nonwhite residents had a higher likelihood of hospitalization, while those in NHs that are part of a chain had a lower likelihood. Furthermore, when the government or a skilled nursing facility operated the nearest hospice, residents had a greater likelihood of hospitalization; they had a lower likelihood if a home health agency operated the nearest hospice.

Our study has some limitations. One is the potential that some relevant confounder was not included, invalidating the unverifiable assumption of ignorable treatment assignment conditional on observables. The heterogeneity of populations and health care practices across facilities, markets, and states presents challenges to adjustments for unobserved factors when estimating population average hospice treatment effect. Finding IVs that satisfy the necessary assumptions across the diverse subpopulations becomes difficult. However, our results illustrate that just increasing the number of observed confounders (and therefore reducing the number of unobserved confounders) in the outcome regression offers some limited adjustment and that it is more important to use some method (like the IPTW method) to restore balance of treatment among individuals with similar observed characteristics. When the IPTW method was used, the importance of adjusting the outcome model for potential confounders became less critical for estimating the hospice treatment effect. In addition, when modeling choice, our analysis had the advantage of the rich NH resident data available in the MDS. Future analysis needs to examine how well our approach performs with community-based hospice patients for which less information is available. Another aspect we have not addressed is the effect of treatment heterogeneity, mostly due to differences in hospice length of stay. Such differences are in part due to the inherent difficulty with determining 6-month terminal prognosis (Christakis 1999; Fox et al. 1999; Christakis and Lamont 2000); and may also be influenced by other barriers to hospice referral in $\mathrm{NHs}$; for example, the absence in many NHs of routine processes for assessing residents' terminal decline, and the physical absence of physicians (Wetle et al. 2005). In our model we do not distinguish hospice treatment by its length of exposure, and, as implemented, the IPTW method obscures the differential effect of this treatment heterogeneity and provides an adjustment that is averaged over the different treatments in the 
sample. An additional concern is the generalizability of our findings to other U.S. states, but previous research (Miller, Gozalo, and Mor 2001) leads us to believe that examination of this phenomenon in other states will result in comparable findings; still, this remains to be seen.

In conclusion, $\mathrm{NH}$ residents enrolled in hospice, versus those dying without hospice, have a lower likelihood of hospitalization in the last 30 days of life. Because of Medicare regulations prohibiting acute care hospitalizations in parallel with hospice enrollment, hospices often intervene when hospitalization is being considered. It appears that this regulation together with the availability of increased end-of-life palliative care management afforded by hospice enrollment are effective in preventing end-of-life hospitalizations.

\section{ACKNOWLEDGMENTS}

We would like to thank Vincent Mor for his guidance in the initial stages of this project, and the reviewers and the Senior Associate Editor for improving the presentation of the manuscript. We would also like to thank the Agency for Healthcare Research and Quality, Department of Health and Human Services, for their financial support (R01 HS10549-01 and R03 HS11457-01).

\section{NOTES}

1. The almost $\$ 3,000$ difference $(\$ 4,445$ among nonhospice versus $\$ 1,551$ among hospice) is statistically significant at any commonly used significance level $(t$-statistic $=11.58$ follows a $t$-distribution with 5,020 degrees of freedom).

2. These states, except Ohio, were selected by CMS (then known as HCFA) as demonstration states to implement a case-mix Medicare system that led to our current PPS Medicare payment system for NHs. As such their MDS data quality is better than in many other states. Ohio was added due to its good MDS data quality and large size to help the power of our analysis.

3. If, for example, 10 individuals share the same value $w$ of observed characteristics $W$ and three of them chose hospice so that $P(H \mid W=w)=0.3$, after IPTW weighting we will have $3 / 0.3=10$ with $H=1$ and $7 / 0.7=10$ with $H=0$.

4. When the probability of treatment varies substantially between subjects it can result in very large variability of the inverse probabilities. Applying these weights directly would make our weighted logistic regression estimates have large variance and fail to be approximately normally distributed. For this reason, we use the stabilized inverse probability weights recommended in the literature defined as the ratio of the unconditional probabilities (proportion in the study with and without hospice) and the conditional probabilities of receiving the treatment actually 
chosen $P / P(W)=P(H=j) / P(H=j \mid W) ; j=0,1$ (Robins 1999; Robins, Hernan, and Brumback 2000).

5. To assess the quality of diagnoses in claims data we compared our diagnosis based on MDS data plus inpatient claims 6 months before death (in hospice equation) or claims before death (in inpatient equation) to those obtained using only MDS data. In the hospice equation, the two methods (MDS+inpatient claims versus MDS alone) had different diagnosis values in 1.6 percent (cancer-no dementia), 1.1 percent (cancer-with dementia), 1.9 percent (dementia-no cancer), and 2.6 percent (other) cases. In the hospitalization equation the mismatch rates were higher (5.1 percent [cancer-no dementia], 5.2 percent [cancer-with dementia], 9.4 percent [dementia-no cancer], and 10.9 percent [other]) due to hospitalizations occurring near death after their last MDS was recorded. In all cases, the errors are among those classified as "other" when using MDS alone moving into cancer or dementia, and those classified as "dementia-no cancer" when using MDS alone moving into cancer-with dementia.

6. The differences are even larger for the 31.6 percent $(N=4,623)$ hospice patients that were enrolled in hospice the entire last 30 days. Their rate was just 1.4 percent.

7. All risk ratios reported were calculated from the estimated OR using the correction method of Zhang and $\mathrm{Yu}$ (1998).

8. Besides noticing that the 95 percent CI of hospice in the two models do not intersect we also formally compared the two models using a Hausman test to check the null hypothesis of no selection on observables bias (Angrist, Imbens, and Rubin 1996). The Hausman test statistic had a value of 106.3 ( $p$-value <.0001) therefore validating the need for controlling for selection bias. We also estimated a conditional logistic regression (equivalent to a fixed effect model that allows a different intercept for each $\mathrm{NH}$ ) to check whether our $\mathrm{NH}$ adjustments were adequately capturing the mean effect of the $\mathrm{NH}$ on hospitalization. The estimates for the hospice and patient-level coefficient were almost identical to those reported on Table 4.

9. We conducted some sensitivity analysis excluding the 15 cancer diagnoses with the lowest average mortality rate (such as prostate, breast, bladder, and leukemia), about 32 percent of all cancer diagnoses. The raw unadjusted hospice effect was now (OR 0.50; [95 percent CI: 0.47, 0.54]) (compared with $0.45[0.42,0.48]$ in Table 3), and using all covariates and the IPTW method yielded $0.59(0.55,0.64)$ (compared with $0.56[(0.53,0.61]$ in Table 3 when using all cancer types).

\section{REFERENCES}

Angrist, J. D., G. W. Imbens, and D. B. Rubin. 1996. "Identification of Causal Effects Using Instrumental Variables." Journal of the American Statistical Association 91: 444-72.

Brooks, S., G. Warshaw, L. Hasse, and J. R. Kues. 1994. "The Physician DecisionMaking Process in Transferring Nursing Home Patients to the Hospital." Archives of Internal Medicine 154: 902-8. 
Centers for Medicare and Medicaid Services. 2001. "Hospice Data-FY 1991 through FY 2001" [accessed October 19, 2002]. Available at http://cms.hhs.gov/ providers/hospiceps/data01.zip

Christakis, N. A. 1999. Death Foretold: Prophecy and Prognosis in Medical Care. Chicago: University of Chicago Press.

Christakis, N. A., and T. J. Iwashyna. 2000. "Impact of Individual Market Factors on the Timing of Initiation of Hospice Terminal Care.” Medical Care 38 (5): 528-41.

Christakis, N. A., and E. B. Lamont. 2000. "Extent and Determinants of Error in Doctors' Prognoses in Terminally Ill Patients: Prospective Cohort Study." British Medical Journal 320 (7233): 469-72.

Cooper, G. S., Z. Yuan, K. C. Stange, S. B. Amini, L. K. Dennis, and A. A. Rimm. 1999. "The Utility of Medicare Claims Data for Measuring Cancer Stage." Medical Care 37 (7): 706-11.

Creditor, M. C. 1993. "Hazards of Hospitalization of the Elderly." Annals of Internal Medicine 118 (3): 219-23.

Diggle, P. J., P. Heagerty, K. Y. Liang, and S. L. Zeger. 2002. Analysis of Longitudinal Data. 2d Edition. New York: Oxford University Press.

Dobalian, A. 2004. "Nursing Facility Compliance with Do-Not-Hospitalize Orders." Gerontologist 44 (2): 159-65.

Emanuel, E. J., and L. L. Emanuel. 1994. "The Economics of Dying. The Illusion of Cost Savings at the End of Life [See Comments]." New EnglandJournal of Medicine 330 (8): 540-4.

Fox, E., K. Landrum-McNiff, Z. Zhong, N. V. Dawson, A. W. Wu, and J. Lynn. 1999. "Evaluation of Prognostic Criteria for Determining Hospice Eligibility in Patients with Advanced Lung, Heart, or Liver Disease. SUPPORT Investigators. Study to Understand Prognoses and Preferences for Outcomes and Risks of Treatments." Journal of the American Medical Association 282 (17): 1638-45.

Fries, B. E., D. P. Schneider, W. J. Foley, M. Gavazzi, R. Burke, and E. Cornelius. 1994. "Refining a Case-Mix Measure for Nursing Homes: Resource Utilization Groups (RUG-III).” Medical Care 32 (7): 668-85.

Gage, B., and T. Dao. 2000. "Medicare's Hospice Benefit: Use and Expenditures 1996 Cohort." In Synthesis and Analysis of Medicare's Hospice Benefit. Washington, DC: U.S. Department of Health and Human Services, Assistant Secretary for Planning and Evaluation, Office of Disability, Aging and Long-Term Care Policy. Available at http://aspe.hhs.gov/daltcp/reports/samhbes.htm

Gallo, W. T., M. J. Baker, and E. H. Bradley. 2001. "Factors Associated with Home versus Institutional Death among Cancer Patients in Connecticut." Journal of the American Geriatrics Society 49 (6): 771-7.

Greer, D. S., V. Mor, S. Sherwood, J. N. Morris, and H. Birnbaum. 1983. "National Hospice Study Analysis Plan." Journal of Chronic Disease 36 (11): 737-80.

Hirano, K., and G. W. Imbens. 2001. "Estimation of Causal Effects Using Propensity Score Weighting." Health Services and Outcomes Research Methodology 2: 259-78.

Hogan, J. W., and T. Lancaster. 2004. "Instrumental Variables and Inverse Probability Weighting for Causal Inference from Longitudinal Observational Studies." Statistical Methods in Medical Research 13: 17-48. 
Horvitz, D. G., and D. J. Thompson. 1952. "A Generalization of Sampling without Replacement from a Finite Universe." Journal of the American Statistical Association 47: $663-85$.

Imbens, G. W. 2004. "Nonparametric Estimation of Average Treatment Effects under Exogeneity: A Review.” Review of Economics and Statistics 86 (1): 4-29.

Intrator, O., N. G. Castle, and V. Mor. 1999. "Facility Characteristics Associated with Hospitalization of Nursing Home Residents: Results of a National Study." Medical Care 37 (3): 228-37.

Kayser-Jones, J. S., C. L. Wiener, and J. C. Barbaccia. 1989. "Factors Contributing to the Hospitalization of Nursing Home Residents." Gerontologist 29: 502-10.

Kidder, D. 1992. "The Effects of Hospice Coverage on Medicare Expenditures.” Health Services Research 27 (2): 195-217.

Lewin-VHI Inc. 1995. An Analysis of the Cost Savings of the Medicare Hospice Benefit. Arlington, VA: National Hospice Organization.

Liang, K. Y., and S. L. Zeger. 1986. "Longitudinal Data Analysis Using Generalized Linear Models." Biometrika 73: 13-22.

McClellan, M. B., and J. P. Newhouse. 2000. "Instrumental Variables Analysis: Applications in Health Services Research.” Health Services Research 35 (5): 1059-202.

Medicare Payment Advisory Commission (MedPac). 2002. Report to Congress: Medicare Beneficiaries' Access to Hospice. Washington, DC: MedPAC.

Miller, S. C., P. L. Gozalo, and V. Mor. 2001. "Hospice Enrollment and Hospitalization of Dying Nursing Home Patients." American Journal of Medicine 111: 38-44.

Miller, S. C., O. Intrator, P. L. Gozalo, J. Roy, J. Barber, and V. Mor. 2004. "Government Expenditures at the End of Life for Short- and Long-Stay Nursing Home Residents: Differences by Hospice Enrollment Status." Journal of the American Geriatrics Society 52 (8): 1284-92.

Miller, S. C., and V. Mor. 2002. "The Role of Hospice Care in the Nursing Home Setting." Journal of Palliative Medicine 5: 271-7.

. 2004. "The Opportunity for Collaborative Care Provision: The Presence of Nursing Home/Hospice Collaborations in the U.S. States.” Journal of Pain and Symptom Management 28 (6): 537-47.

Mor, V., O. Intrator, B. E. Fries, C. Phillips, J. Teno, C. Hawes, and J. Morris. 1997. "Changes in Hospitalization Associated with Introducing the Resident Assessment Instrument." Journal of the American Geriatrics Society 45 (8): 1002-10.

Mor, V., and D. Kidder. 1985. "Cost Savings in Hospice: Final Results of the National Hospice Study." Health Services Research 20 (4): 407-22.

Mor, V., G. Papandonatos, and S. C. Miller. 2005. "End-of-Life Hospitalization for African American and Non-Latino White Nursing Home Residents: Variation by Race and Facility's Racial Composition.” Journal of Palliative Medicine 8 (1): $58-68$.

Morris, J. N., B. E. Fries, D. R. Mehr, C. Hawes, C. Phillips, V. Mor, and L. A. Lipsitz. 1994. “MDS Cognitive Performance Scale.” Journal of Gerontology 49 (4): M174-82.

Petrisek, A. C., and V. Mor. 1999. "Hospice in Nursing Homes: A FacilityLevel Analysis of the Distribution of Hospice Beneficiaries." Gerontologist 39 (3): 279-90. 
Pritchard, R. S., E. S. Fisher, J. M. Teno, S. M. Sharp, D. J. Reding, W. A. Knaus, J. E. Wennberg, and J. Lynn. 1998. "Influence of Patient Preferences and Local Health System Characteristics on the Place of Death." Journal of the American Geriatrics Society 46: 1242-50.

Robins, J. M. 1999. "Marginal Structural Models versus Structural Nested Models as Tools for Causal Inference.” In Statistical Models in Epidemiology: The Environment and Clinical Trials, edited by M. Halloran and D. Berry, pp. 95-134. New York: Springer-Verlag.

Robins, J. M., M. A. Hernan, and B. Brumback. 2000. "Marginal Structural Models and Causal Inference in Epidemiology.” Epidemiology 11 (5): 550-60.

Robins, J. M., and A. Rotnitzky. 1995. "Semiparametric Efficiency in Multivariate Regression Models with Missing Data." Journal of the American Statistical Association 90: 122-9.

Saliba, D., R. Kington, J. Buchanan, R. Bell, M. M. Wang, M. Lee, M. Herbst, D. Lee, D. Sur, and L. Rubenstein. 2000. "Appropriateness of the Decision to Transfer Nursing Facility Residents to the Hospital." Journal of the American Geriatrics Society 48 (2): 154-63.

Scitovsky, A. A. 1994. "The High Cost of Dying Revisited.” Milbank Quarterly 72 (4): 561-91.

Wetle, T., R. S. Shield, J. Teno, S. C. Miller, and L. Welch. 2005. "Family Perspectives on End-of-Life Care Experiences in Nursing Homes." Gerontologist 45: 642-50.

Zhang, J., and K. F. Yu. 1998. "What's the Relative Risk? A Method of Correcting the Odds Ratio in Cohort Studies of Common Outcomes." Journal of the American Medical Association 280: 1690-1.

Zweig, S. C., R. L. Kruse, E. F. Binder, K. L. Szafara, and D. R. Mehr. 2004. "Effect of Do-Not-Resuscitate Orders on Hospitalization of Nursing Home Residents Evaluated for Lower Respiratory Infections.” Journal of the American Geriatrics Society 52 (1): 51-8. 